Artículo de Investigación, Científica y Tecnológica

Recepción: 13 de junio de 2019

Aprobación: 26 de junio de 2021

\title{
EXPRESIONES DE DESIGUALDAD EDUCATIVA EN COLOMBIA: UNA REFLEXIÓN DESDE LOS INDICADORES DE CONTEXTO, ACCESO Y RESULTADOS
}

\section{EXPRESSIONS OF EDUCATIONAL INEQUALITY IN COLOMBIA: A REFLECTION FROM THE CONTEXT, ACCESS AND RESULTS INDICATORS \\ EXPRESSÕES DA DESIGUALDADE EDUCACIONAL NA COLÔMBIA: UMA REFLEXÃO DOS INDICADORES DE CONTEXTO, ACESSO E RESULTADOS}

\section{Aracely Burgos-Ayala'}

Anderson Geovany Rodríguez Buitrago²

\section{¿Cómo citar este artículo?:}

Burgos-Ayala, A. y Rodríguez Buitrago, A. G. (2021). Expresiones de desigualdad educativa en Colombia: una reflexión desde los indicadores de contexto, acceso y resultados. Cultura Científica, 19. 19, pp.59-80.

https://doi.org/10.38017/1657463X.735

1 MSc. en Ciencias, Universidad de Chile. Docente, Fundación Universitaria Juan de Castellanos. aburgosa@jdc.edu.co.

2 MSc. en Docencia e Investigación Universitaria, Universidad Sergio Arboleda. Docente, Fundación Universitaria Juan de Castellanos. arodriguez@jdc.edu.co. 


\section{RESUMEN}

La desigualdad educativa es una de las reproducciones sociales más reiteradas en América Latina (AL). En Colombia, el segundo país más desigual de $A L$, la desigualdad educativa persiste a pesar de las recientes políticas educativas. Se analizaron las tendencias de desigualdad en educación obligatoria en Colombia respecto a los indicadores de contexto, acceso y resultados en: 1) Sistema Educativo incluido en el informe de la Organización para la Cooperación y el Desarrollo Económico (año 2016); y, 2) Sistema de Información de Tendencias Educativas en América Latina (años 2010, 2015, 2016 y 2018). Para la OCDE, la pobreza y desigualdad se convierten en retos significativos, que inciden positiva o negativamente en los indicadores analizados y en la calidad educativa. Para el SITEAL, se encontró que cerca del $10 \%$ de las personas aún no asisten al sistema educativo, y de los que logran acceder, el egreso en la básica secundaria no supera al $80 \%$; el acceso tiende a incrementar en el tiempo, aunque es mayor cuando los ingresos económicos son más altos. Se evidencia que las mujeres asisten y egresan más al sistema educativo que los hombres, esto demuestra que la desigualdad educativa se relaciona íntimamente con aspectos sociales y demográficos. La pobreza sigue siendo causa de exclusión educativa. Se insta a investigar más detalladamente estos indicadores en contextos más focalizados para descubrir explicaciones más detalladas y posibles oportunidades emergentes localizadas frente esta desigualdad.

Palabras clave: indicadores educativos, desigualdad educativa, contexto, acceso, resultados.

\section{ABSTRACT}

Educational inequality is one of the most reiterated social reproductions in Latin America (LA). In Colombia, the second most unequal country in LA, educational inequality persists despite recent educational policies. Inequality trends in compulsory education in Colombia were analyzed concerning context, access, and results from indicators in 1) Education System included in the report of the Organization for Economic Cooperation and Development (the year 2016); and, 2) Information System of Educational Trends in Latin America (years 2010, 2015, 2016 and 2018). For the OECD, poverty, and inequality become significant challenges, which have a positive or negative impact on the indicators analyzed and on educational quality. For SITEAL (Sistema de Información de Tendencias Educativas en América Latina- Information System on Educational Trends in Latin 
America), it was found that about $10 \%$ of people still do not attend the education system, and of those who manage to access, the graduation in basic secondary education does not exceed $80 \%$; access tends to increase over time, although it is higher when the economic income get bigger. It is evident that women attend and leave the educational system more than men, which shows that educational inequality is closely related to social and demographic aspects. Poverty continues to be a cause of educational exclusion. Further research on these indicators in more focused contexts is called for to discover more detailed explanations and possible localized emerging opportunities in the face of this inequality.

Keywords: educational indicators, educational inequality, context, access, outcomes.

\section{RESUMO}

A desigualdade educacional é uma das reproduções sociais mais repetidas na América Latina $(\mathrm{AL})$. Na Colômbia, o segundo país mais desigual da AL, a desigualdade educacional persiste, apesar das políticas educacionais recentes. As tendências de desigualdade na escolaridade obrigatória na Colômbia foram analisadas com relação aos indicadores de contexto, acesso e resultados em: 1) Sistema Educacional incluído no relatório da Organização para a Cooperação e Desenvolvimento Econômico (ano 2016); e, 2) Sistema de Informação sobre Tendências Educacionais na América Latina (anos 2010, 2015, 2016 e 2018). Para a OCDE, a pobreza e a desigualdade tornam-se desafios significativos, com impacto positivo ou negativo nos indicadores analisados e na qualidade educacional. Para o SITEAL, constatou-se que cerca de $10 \%$ das pessoas ainda não frequentam a rede de ensino e, das que conseguem acessar, a conclusão do ensino médio não passa de $80 \%$; o acesso tende a aumentar com o tempo, embora seja maior quando a renda é maior. É evidente que as mulheres frequentam e se formam mais no sistema educacional do que os homens, o que mostra que a desigualdade educacional está intimamente relacionada aos aspectos sociais e demográficos. A pobreza continua a ser uma causa de exclusão educacional. Uma investigação mais aprofundada desses indicadores em contextos mais específicos é encorajada para descobrir explicações mais detalhadas e potenciais oportunidades emergentes localizadas para esta desigualdade.

Palavras-chave: indicadores educacionais, desigualdade educacional, contexto, acesso, resultados. 
La educación permite evidenciar tanto la desigualdad en la sociedad, como revertir

la reproducción de otras desigualdades estructurales, como la pobreza, que afectan el bienestar de los individuos

\section{INTRODUCCIÓN}

La educación permite evidenciar tanto la desigualdad en la sociedad, como revertir la reproducción de otras desigualdades estructurales, como la pobreza, que afectan el bienestar de los individuos (Cecchini et al., 2014). A través de la educación, es posible pensar en el futuro; esta se convierte en la posibilidad de otra historia (Serra y Canciano, 2006). En este sentido, han transcurrido más de 70 años de la declaración "toda persona tiene derecho a la educación” (Asamblea General de las Naciones Unidas, 1948, art. 26) que sigue vigente, por ejemplo, en el objetivo 4 y 10 de la Agenda de Desarrollo Sostenible, como una herramienta para la reducción de la desigualdad propuesta. La búsqueda de igualdad educativa hace parte de la demanda de justicia social, por lo que no es un producto natural del orden social, sino el resultado de acciones conscientes, voluntarias, reflexivas (Giddens, citado en Tedesco, 2010). De forma particular, para América Latina (AL), Gentili (2009) explica que en la educación existen complejos procesos y dificultades de consolidación como un derecho humano.

Al persiste como una de las regiones más inequitativas a nivel mundial (Trucco, 2014), a pesar de que allí han ocurrido importantes transformaciones de políticas sociales en últimas décadas. Ejemplo de esto han sido las políticas relacionadas con la educación (Cecchini et al., 2014), que han buscado moderar o disminuir las inequidades (Atorresi,
Mekler, y Feldman, 2012). Este panorama sugiere que los sistemas escolares de AL muestran mejoras evidentes (cita), pero persistan determinadas limitaciones (OECD, United Nations y CAF, 2014). Algunos indicadores que componen el sistema educativo como los son los de contexto, acceso y resultados, hacen parte de dichas limitaciones. La integración de estos indicadores, podrían dar luces de realidades de desigualdad educativa.

El contexto permite "caracterizar las condiciones demográficas, sociales, económicas y culturales en las que se desarrolla la educación en Colombia" (Ministerio de Educación, 2013). El acceso evidencia los niveles de escolarización obligatoria en diversas franjas de edad (temprana 3 a 5 años, adolescencia 13 a 17 años, que refiere a la secundaria) (Bellei et al., 2012; Bellei et al., 2013; Bolívar, 2005)particularmente en las teorías de la justicia distributiva. Éstas aunque diversas y plurales, y con mayor apoyo y legitimidad unas que otras, pueden servir de base para evaluar su equidad o inequidad. No obstante, si bien todas las teorías coinciden en la igualdad, el asunto en que divergen se relaciona con la siguiente pregunta: ¿igualdad de qué?, según la conocida formulación de Sen (1995. El acceso también se expresa en la tasa de matrícula y retención (Cecchini et al., 2014) y en el aumento de la esperanza de vida escolar, p "permanencia" (Cecchini et al., 2014; Gentili, 2009). Los resultados, por su parte, "permiten realizar un análisis sobre el logro y 
calidad del sistema educativo, medido a través del resultado escolar de los alumnos mediante las pruebas de evaluación, la graduación, la certificación” (MinEducación, 2013, p. 22). Los resultados se han asociado al número de estudiantes que egresa o se gradúa, así como la disminución de la tasa de analfabetismo (Gentili, 2009).

Una mirada local de AL se puede hacer en Colombia, segundo país más inequitativo en ingresos y en desigualdad en educación en todo el continente (PNUD, 2015). Allí, al igual que $A L$, se ha avanzado en políticas educativas (Congreso de la República de Colombia, 1994, 2001, 2013; Departamento Nacional de Planeación, 2007; Ministerio de Educación Nacional, 2006; Ministerio de Educación Nacional, 2003, 2006; Presidencia de la República de Colombia, 2011, 2017, 1978, 1997, 2002, entre otros). Estas podrían surgir como respuesta a la Declaración Universal de los Derechos Humanos (Asamblea General de las Naciones Unidas, 1948), a la Constitución Política de 1991 (Asamblea Nacional Constituyente, 1991), y más recientemente a la Agenda 2030 de Desarrollo Sostenible (ONU, 2015), que buscan el logro de diversos derechos sociales.

Este país ha tenido importantes logros en los diversos indicadores de contexto, acceso y resultados educativos, pero persisten retos por abordar. Avances como el aumento en la inversión del Producto Interno Bruto (Ministerio de Educación Cultura y Deporte, 2014) han impactado en la obligatoriedad de la educación entre los 5 y los 15 años (Ministerio de Educación Nacional/ OCDE, 2016). Sin embargo, allí el acceso a la etapa inicial, decisiva en la vida de las personas, es reducida en comparación con otros países (Ministerio de Educación Cultura y Deporte, 2014). En tal sentido, en el presente artículo se pretende explorar cómo han ocurrido tendencias de expresiones de desigualdad educativa en Colombia a partir de los indicadores de contexto, acceso y resultados del sistema educativo, teniendo como referente el informe del Ministerio Nacional de Educación/OCDE (Ministerio de Educación Nacional/OCDE, 2016) y el Sistema de Información de Tendencias Educativas en América Latina (años 2010, 2015, 2016, 2017, 2018).

\section{METOdOLOGÍA}

\subsection{Colecta de datos}

Para el logro del objetivo, se recurrió a los datos reportados en el informe del Ministerio de Educación Nacional (MEN)/OCDE (Ministerio de Educación Nacional/OCDE, 2016), y en el Sistema de indicadores de Información de Tendencias Educativas en América Latina para los años 2010, 2015, 2016, 2017, 2018. La OCDE agrupa 36 países, promueve políticas, como la educativa, que mejoran el bienestar económico y social de las personas alrededor del mundo (Ministerio de Educación Nacional/OCDE, 2016). El SITEAL, por su parte, ha tenido presencia en 20 países por más de 25 años y es una plataforma coordinada por 
la UNESCO, IIPE y la OEI. Ambas recopilan e integran datos relacionados con aspectos sociales y educativos en América Latina y el Caribe.

$\mathrm{El}$ documento "Indicadores en Educación" (UNESCO, 2009) y el "Sistema Nacional de Indicadores educativos para los niveles de preescolar, básicas y media en Colombia" (Ministerio de Educación, 2013) guiaron las especificaciones técnicas, alcances y limitaciones de los indicadores. Se recurrió a indicadores por ser instrumentos que proporcionan información relevante acerca de algún aspecto significativo de la realidad (Botinelli, 2016). Se optó por el uso de indicadores como una de las formas de analizar la desigualdad educativa. Los indicadores educativos son aceptados como herramientas que permiten captar aspectos de una realidad social (Tiana, 1997), con la posibilidad de permitir comparar y juzgar el contexto, el funcionamiento de la enseñanza, y sus resultados (Morduchowicz, 2006). Las categorías permitieron delimitar la revisión de aspectos puntuales en cada indicador, recopilando y usando datos, con el fin de orientar futuras acciones de mejora en los contextos educativos, y permitiendo identificar áreas en las que necesita desarrollarse más. La Tabla 1 resume los indicadores, categorías y sub-categorías de análisis tanto para OCDE como para el SITEAL.

Tabla 1. Indicadores, categorías y sub-categorías de análisis tanto para OCDE como para el SITEAL.

\begin{tabular}{|c|c|c|c|c|c|c|}
\hline Indicador & Categoría & Sub-categoría & & aálisis OCDE & & Análisis SITEAL* \\
\hline \multirow{2}{*}{ Contexto * * } & Demográfico & Sexo & \multirow{2}{*}{ 1) } & \multirow{2}{*}{$\begin{array}{l}\text { Pobreza y } \\
\text { desigual- } \\
\text { dad. }\end{array}$} & \multirow[b]{2}{*}{ 1) } & \multirow[b]{2}{*}{$\begin{array}{l}\text { Tasa de asistencia } \\
\text { escolar por sexo }\end{array}$} \\
\hline & Sociales & $\begin{array}{l}\text { Nivel de } \\
\text { ingresos }\end{array}$ & & & & \\
\hline Acceso & Asistencia & $\begin{array}{c}\text { Tasa de asistencia } \\
\text { escolar }\end{array}$ & & \multirow{2}{*}{$\begin{array}{l}\text { Ingreso tar- } \\
\text { dío y altos } \\
\text { índices de } \\
\text { repetición } \\
\text { por grados. } \\
\text { Permanen- } \\
\text { cia y conti- } \\
\text { nuidad. } \\
\text { Resultados } \\
\text { pruebas } \\
\text { PISA. }\end{array}$} & 2) & $\begin{array}{l}\text { Tasa de asistencia } \\
\text { escolar por niveles } \\
\text { de ingresos } \\
\text { Tasa de finalización } \\
\text { de la educación se- } \\
\text { cundaria por sexo }\end{array}$ \\
\hline Resultados & Egreso & $\begin{array}{c}\text { Tasa de } \\
\text { finalización } \\
\text { de la educación } \\
\text { secundaria }\end{array}$ & 3) & & 4) & $\begin{array}{l}\text { Tasa de finalización } \\
\text { de la educación se- } \\
\text { cundaria por sexo } \\
\text { Tasa de finalización } \\
\text { de la educación se- } \\
\text { cundaria por niveles } \\
\text { de ingresos }\end{array}$ \\
\hline
\end{tabular}

* Años 2010, 2015, 2016, 2017, 2018.

** Indicador que interactúa con todos los demás indicadores.

Nota: se trata de una elaboración propia basada en "Revisión de políticas nacionales de educación. La educación en Colombia”, por Ministerio de Educación Nacional/OCDE, 2016, https://bit.ly/3eFuuHD, y los informes SITEAL para los años 2010, 2015, 2016, 2017 y 2018. 


\subsection{Análisis de datos}

Se hace un análisis exploratorio descriptivo para identificar brechas, por lo tanto, no hay un abordaje exhaustivo multidimensional de la desigualdad educativa en Colombia.

\section{RESULTADOS DISCUSIÓN}

\subsection{Desde la OCDE: Contexto}

Las transiciones en Colombia dependen de distintos factores, pero ninguno será más importante para el futuro, que la capacidad de ir logrando un sistema educativo sólido. Por lo tanto, al mirar el primer indicador referente al contexto, es necesario considerar la pobreza y desigualdad como retos prioritarios para Colombia, esto es aún más notorio cuando se realiza una comparación entre regiones, "aproximadamente uno de cada tres colombianos vive en condiciones de pobreza $(33 \%)$, una cifra mayor a la de cualquier país de la OCDE y considerablemente superior al promedio de la OCDE del $11 \%$ " (Ministerio de Educación Nacional/OCDE, 2016).

La pobreza y desigualdad, conllevan a escenarios de discriminación, inequidades, falta de oportunidades, poco reconocimiento y disminución de condiciones de posibilidad frente al acceso y permanencia en los procesos educativos. Igualmente, tiene que ver con los logros esperados y los objetivos que se pueden alcanzar, tanto individuales como colectivos. Al respecto, el informe del MEN y la OCDE (Ministerio de Educación
Nacional/OCDE, 2016) indican que los estudiantes menos favorecidos en cuanto a recursos, cuentan con desventajas en los procesos de formación, "la evidencia proveniente de un análisis de las pruebas SABER indica que el nivel socioeconómico de las instituciones educativas explica en gran parte la diferencia en cuanto a los logros" (Duarte et al., 2014, y Delgado, 2014, citados en Ministerio de Educación Nacional/ OCDE, 2016, p. 36). La tasa de segregación es mayor dependiendo del contexto socioeconómico donde se encuentre ubicada la Institución Educativa, esto puede generar efectos negativos en el desempeño académico de los estudiantes.

El panorama es más desolador cuando se revisan las diferencias entre el sector rural y urbano. En este aspecto, es importante señalar que el país es diverso y existen condiciones complejas que conllevan a desigualdades económicas y educativas vinculadas con lo económico y estructural. "Los colombianos que viven en zonas rurales tienen múltiples desventajas, como mayores índices de pobreza ( $43 \%$ en comparación con el $\mathbf{2 7 \%}$ en zonas urbanas), desnutrición, embarazo de adolescentes y violencia, sumado a una infraestructura insuficiente" (CIPI, citado en Ministerio de Educación Nacional/OCDE, 2016, p. 36). En las distintas regiones de Colombia, las tasas de pobreza son mucho más evidentes en zonas rurales, esto se convierte en tema para analizar para el sistema educativo; por ejemplo, en las comunidades indígenas y afrocolombianos,

\author{
"Los \\ colombianos \\ que viven en zonas \\ rurales tienen \\ múltiples \\ desventajas, \\ como mayores \\ índices de \\ pobreza $(43 \%$ en \\ comparación con \\ el $\mathbf{2 7} \%$ en zonas \\ urbanas), \\ desnutrición, \\ embarazo de \\ adolescentes y \\ violencia, \\ sumado a una \\ infraestructura \\ insuficiente"
}


Es necesaria una educación más pertinente de acuerdo con las necesidades de cada región, de esta manera la educación se convierte en algo significativo para seres humanos de distintos contextos sociales y culturales, y con diferentes capacidades e intereses la mayoría viven en zonas rurales, y enfrentan barreras adicionales para acceder a la educación (Delgado, 2014).

Por tanto, es necesaria una educación más pertinente de acuerdo con las necesidades de cada región, de esta manera la educación se convierte en algo significativo para seres humanos de distintos contextos sociales y culturales, y con diferentes capacidades e intereses. La pertinencia conlleva a que lo que se enseña y aprende estará orientado a responder a un contexto y circunstancias específicas, pero sin perder la mirada en la interacción con un mundo global; asimismo, promueve el desarrollo de potencialidades, actitudes y aptitudes que permitan desarrollar competencias para superar la pobreza y desigualdad. "Realizar más esfuerzos de manera proactiva para compartir y multiplicar las innovaciones y buenas prácticas locales permitiría agilizar las mejoras en todo el sistema" (Ministerio de Educación Nacional/OCDE, 2016).

\subsection{Desde la OCDE: Acceso}

Respecto al acceso, el MEN y la OCDE (2016) evidencian cómo las políticas educativas han generado un incremento progresivo en la ampliación y participación dentro del sistema educativo colombiano, especialmente, en los niveles de Preescolar, Secundaria y Educación Superior. Entre 2000 y 2012-2013, la proporción de niños matriculados en educación preescolar (es decir, los programas para niños menores de la edad de ingreso a primaria, pero con un componente educativo) "se incrementó del $36 \%$ al 45 $\%$, una cifra aún inferior al promedio de la OCDE (84 \%) pero superior a Turquía (28\%) (UNESCO-UIS, 2015)" (Ministerio de Educación Nacional/OCDE, 2016).

Estos datos del MEN y la OCDE (2016) son fundamentales, teniendo presente que el aumento en el acceso a la primera infancia se convierte en una oportunidad para combatir los círculos de pobreza, y en una posibilidad para construir una sociedad más equitativa. Este incremento se evidencia también en la educación básica y media. Una serie de medidas, desde la prestación de educación pública gratuita hasta grandes inversiones en infraestructura y recursos educativos, han permitido ampliar drásticamente la cobertura de educación secundaria. "Entre 2002 y 2012 2013, las tasas netas de matriculados aumentaron del $59 \%$ al $70 \%$ en básica secundaria y del $30 \%$ al $41 \%$ en educación media (UNESCO-UIS, 2015)" (Ministerio de Educación Nacional/OCDE, 2016).

El número de inscritos en educación superior también se ha acrecentado, "con una tasa bruta de matriculados duplicada, del $24 \%$ al $48 \%$ entre 2000 y 2013 . El promedio de la OCDE aumentó del $52 \%$ al $72 \%$ durante el mismo período (UNESCO-UIS, 2015, p. 29)" (Ministerio de Educación Nacional/OCDE, 2016). Con este panorama, Colombia necesita seguir aumentando la participación en la educación superior, mejorando los préstamos y los sistemas de becas y ampliando 
el número de cupos de la manera más equilibrada posible por todo el país. El informe MEN/OCDE (2016) señala que es necesario establecer una línea de reflexión sobre la permanencia y continuidad en los grados posteriores; esto es fundamental partiendo de la premisa de que el hombre, como ser social, necesita de manera prospectiva estar en permanente relación con los otros y con todo lo que le rodea. En este contacto, se hace partícipe de la diversidad de pensamientos, sentimientos, necesidades, ritmos y estilos de aprendizaje, comportamientos, tradiciones y acciones dentro de la dinámica de los grupos sociales; por tanto, es importante interpelarnos sobre la relación entre el incremento en el acceso, la esperanza de vida escolar y la adecuada transición entre los niveles de educación.

El informe mencionado refiere que, pese a que se evidencia un incremento en la permanencia y continuidad en el Sistema educativo, "solo los niños con edades entre 7 y 13 años están cerca de disfrutar de acceso universal a la educación (definido como tasas de inscritos superiores al $90 \%$ )" (Ministerio de Educación Nacional/OCDE, 2016, p. 29). Esto es necesario revisarlo en comparación con la mayoría de los países de la OCDE, quienes aseguran acceso universal o un alto número de inscritos para todos los niños entre 5 y 14 años (Ministerio de Educación Cultura y Deporte, 2014). Son varios los factores que inciden en las bajas tasas de asistencia escolar en Colombia, entre otros:
Una deficiente transición entre niveles, las altas tasas de deserción escolar y un número significativo de niños que no ingresan al sistema educativo. Aunque la tasa de deserción desde preescolar hasta educación secundaria se ha reducido a más de la mitad entre 2002 y 2013 , la proporción de niños en edad de cursar primaria que no están estudiando, de hecho, ha aumentado, del 4 $\%$ en 2000 al $9 \%$ en 2010 . Aproximadamente, uno de cada cinco estudiantes en Colombia no continúa estudiando después de la primaria (OCDE/CAF/CEPAL, 2014). (Ministerio de Educación Nacional/OCDE, 2016, p. 29)

A estas situaciones, asociadas con el acceso y participación de los estudiantes colombianos en la educación, debe sumarse el ingreso tardío y los altos índices de repetición por grados. Actualmente, se evidencian algunos esfuerzos que, con el tiempo, pueden generar impacto dentro de las políticas educativas; por ejemplo, a través del Índice de Calidad Educativa (ISCE). Sin embargo, dentro del informe MEN/ OCDE (2016) se mencionan estas dos situaciones como impulsadoras de los bajos logros académicos y el aumento en el riesgo de deserción. En lo que refiere al ingreso tardío, el Ministerio de Educación Nacional/ OCDE (2016) muestra que es común que un alto número de estudiantes no se encuentran matriculados en el grado que corresponde de acuerdo con su edad, "aunque el índice bruto de niños matriculados entre los niños de 5 años en Colombia es del $89 \%$, solo el $57 \%$ están en el grado apropiado (el año de transición), lo cual está 20 puntos 
Colombia tiene uno de los porcentajes más altos de jóvenes entre los 15 y los 19 años de edad que no están estudiando; un $36 \%$ en total, es decir, más del doble que el promedio de la OCDE porcentuales por debajo del promedio de la OCDE" (Ministerio de Educación Nacional/OCDE, 2016, p. 31). El panorama es similar en los estudiantes que cursan los grados de básica primaria, siguiendo el informe del MEN/OCDE (2016) “el 84 $\%$ de los estudiantes están matriculados en el grado correspondiente a su edad, frente a una Tasa Bruta de Matrícula del $107 \%$, que es la brecha más grande entre los países de la OCDE” (p. 31).

Referente a la repetición de grados en Colombia, el indicador es uno de los más altos entre los países que participan en el Programa Internacional para la Evaluación de Estudiantes (PISA) de la OCDE: "en 2012, el $41 \%$ de los jóvenes de 15 años había repetido por lo menos un grado, en comparación con el promedio de la OCDE del 12\% (OCDE, 2013b)" (Ministerio de Educación Nacional/OCDE, 2016, p. 32).

La alta tasa de repetición de grados es un elemento importante en el momento de buscar las causas a la proporción de estudiantes demasiado mayores para los grados que cursan. Esto puede conllevar a una disminución en el rendimiento académico de los estudiantes de mayor edad, baja autoestima, carencia de sentido frente a los procesos de formación, generando un aumento en la deserción, "además, los estudiantes mayores son los que pagan los costos de oportunidad más altos por permanecer en el sistema educativo (OCDE, 2012a)" (Ministerio de Educación Nacional/OCDE, 2016).
La educación superior no está fuera de esta realidad, por el contrario, es una continuación. El riesgo es que se transforme en ser un residual o intento de solucionar lo que los ciclos anteriores no han hecho. En concordancia, encontramos en el informe del MEN/OCDE (2016) que las tasas de deserción anual en educación superior varían entre el 10,4 $\%$ en las universidades y el $22,2 \%$ en las instituciones técnicas y tecnológicas. Estas constantes tasas de deserción y la débil transición, son indicadores a tener presentes, ya que "Colombia tiene uno de los porcentajes más altos de jóvenes entre los 15 y los 19 años de edad que no están estudiando; un $36 \%$ en total, es decir, más del doble que el promedio de la OCDE (13\%)" (Ministerio de Educación Nacional/ OCDE, 2016, p. 30).

\subsection{Desdela OCDE: Resultados}

En lo relacionado con el indicador de resultados, se puede pensar en el Programa para la Evaluación Internacional de Alumnos de la OCDE (PISA, por sus siglas en inglés), como uno de los indicadores que han sido referentes en el momento de analizar la calidad en educación, estas pruebas permiten evaluar qué saben y qué pueden hacer con lo que saben los estudiantes de 15 años en todo el mundo. En el caso de Colombia, el país ha participado en estas pruebas desde el año 2006. Es pertinente recordar que PISA permite referenciar aquellos países que han venido alcanzando un buen rendimiento, influenciado por la repartición equitativa de oportunidades en el contexto y acceso al aprendizaje. 
En el informe del MEN/OCDE (2016), se plasman algunos datos importantes relacionados con este aspecto: "Los resultados sugieren que la comprensión lectora de los estudiantes ha mejorado desde los primeros ciclos de evaluación hasta el presente, pero sigue siendo baja comparada con el promedio de la OCDE y con otros países latinoamericanos" (p. 32). El desempeño no ha variado en las áreas de matemáticas y ciencias. "En matemáticas, los estudiantes colombianos de 15 años están, en promedio, atrasados más de tres años (118 puntos) con respecto a sus pares de países miembros de la OCDE (OCDE, 2014)" (Ministerio de Educación Nacional/OCDE, 2016).

Esta reflexión sobre los resultados en las pruebas se debe suscitar de manera transversal desde los primeros años de formación de los estudiantes, ya que, como lo menciona el Tercer Estudio Regional Comparativo y Explicativo (TERCE), encargado de evaluar las competencias en ciencias, matemáticas y escritura de los estudiantes latinoamericanos de los Grados 3 y 6; se evidencia que "los estudiantes colombianos empiezan a atrasarse con respecto a sus países vecinos como Chile, Costa Rica y México, en los primeros años de educación (Oficina de la UNESCO de Santiago, 2015)" (Ministerio de Educación Nacional/OCDE, 2016). Este panorama es similar al que se encuentran en las pruebas nacionales SABER en grado 9 y grado 11 , "también muestran niveles globales bajos, con tendencias negativas en los puntajes de lectura y matemáticas en algunos grados y años (Ministerio de Educación Nacional/ OCDE, 2016).

Colombia ha venido progresando desde el año 2006 en los indicadores de las pruebas PISA, especialmente en los desempeños relacionados con ciencia, matemáticas y lectura; sin embargo, la proporción de estudiantes con menores desempeños sigue siendo una de las más altas dentro del informe del MEN (2016):

Los estudiantes con el desempeño más bajo en lectura (décimo percentil más bajo) mejoraron sus puntajes en las pruebas PISA en más de 50 puntos hasta llegar a 295 puntos entre 2006 y 2012 , equivalentes a un poco más de un año escolar. (Ministerio de Educación Nacional/OCDE, 2016)

No obstante, se constata que la proporción de estudiantes con menor rendimiento no disminuyó significativamente en los ciclos de las pruebas PISA, y esto podría extenderse a ciclos anteriores de formación, especialmente en las evaluaciones de lectura y escritura de las pruebas nacionales SABER 2014 , donde "se encontró que el $49 \%$ de los estudiantes en Grado 3, el $67 \%$ en Grado 5 y el 73 \% en Grado 9 no cumplían los estándares mínimos" (Ministerio de Educación Nacional/ OCDE, 2016, p. 33).

Esto lleva a pensar en la preparación adecuada de los estudiantes colombianos en el momento del ingreso a la educación superior; escenario en el que la inequidad, exclusión y baja calidad de la formación,

\footnotetext{
"En matemáticas, los estudiantes colombianos de 15 años están, en promedio, atrasados más de tres años (118 puntos) con respecto a sus pares de países miembros de la OCDE.
} 
pueden estar asociadas a las competencias con las que egresan los estudiantes de los ciclos anteriores. Igualmente, es menester revisar las formas de evaluación general, las cuales están orientadas a observar el país de manera igualitaria, como si al parecer, las condiciones de posibilidad educativa fueran similares para todos.

En las pruebas PISA 2006, el 56 $\%$ de los estudiantes colombianos de 15 años tuvo un desempeño inferior al nivel 2 de aptitud, comparado con el promedio de la OCDE del $21 \%$. En las pruebas PISA 2012, estadísticamente la proporción permaneció igual, en $51,4 \%$, muy por encima del promedio de la OCDE del $18 \%$ (OCDE, 2014b). (Ministerio de Educación Nacional/OCDE, 2016)

Al finalizar los ciclos de la educación básica y media, cuando los estudiantes terminan y van a los niveles de educación superior, los niveles de competencia de unos y otros son inferiores, por ejemplo, revisando la media correspondiente al contexto europeo. "Los sistemas de información-que son sólidos, pero están subutilizados en Colombia- deben reorientarse hacia el mejoramiento y deben fortalecerse para enfocarse en lo que importa: brindar oportunidades de aprendizaje de buena calidad para todos" (Ministerio de Educación Nacional/OCDE, 2016). Análogamente, cabe preguntarse: ¿cuáles son las oportunidades en términos de calidad en este contexto?, ¿cómo generar competencias cuando se están transformando los contextos laborales?, ¿de qué forma pueden las Universidades responder a estas situaciones?

\subsection{Desde el SITEAL: Contex- to y Acceso}

Los datos del SITEAL revelan, de forma general, una tendencia al aumento el acceso entre 2010 y 2017 , existiendo una pequeña, pero mayor tendencia, para las mujeres. No obstante, al 2018 se observa una leve disminución tanto en hombres como en mujeres (figura 1A). Esto implica que, al menos para el acceso, hay una tendencia a lograr las expectativas normativas nacionales, resultado que podría atribuires a las diversas iniciativas políticas que se han propuesto para hacer frente a la desigualdad educativa (Frigerio, Diker y Mendoza, 2009). No obstante, la tasa de escolarización aún no se cumple en su totalidad. 
Figura 1. Indicadores de Contexto/Acceso: A). Tasa de asistencia escolar por sexo (M=Masculino; F=Femenino); B). Tasa de asistencia escolar por nivel de ingresos. Indicadores de Contexto/Resultados: C). Tasa de finalización de la educación secundaria por sexo; D). Tasa de finalización de la educación secundaria por nivel de ingresos

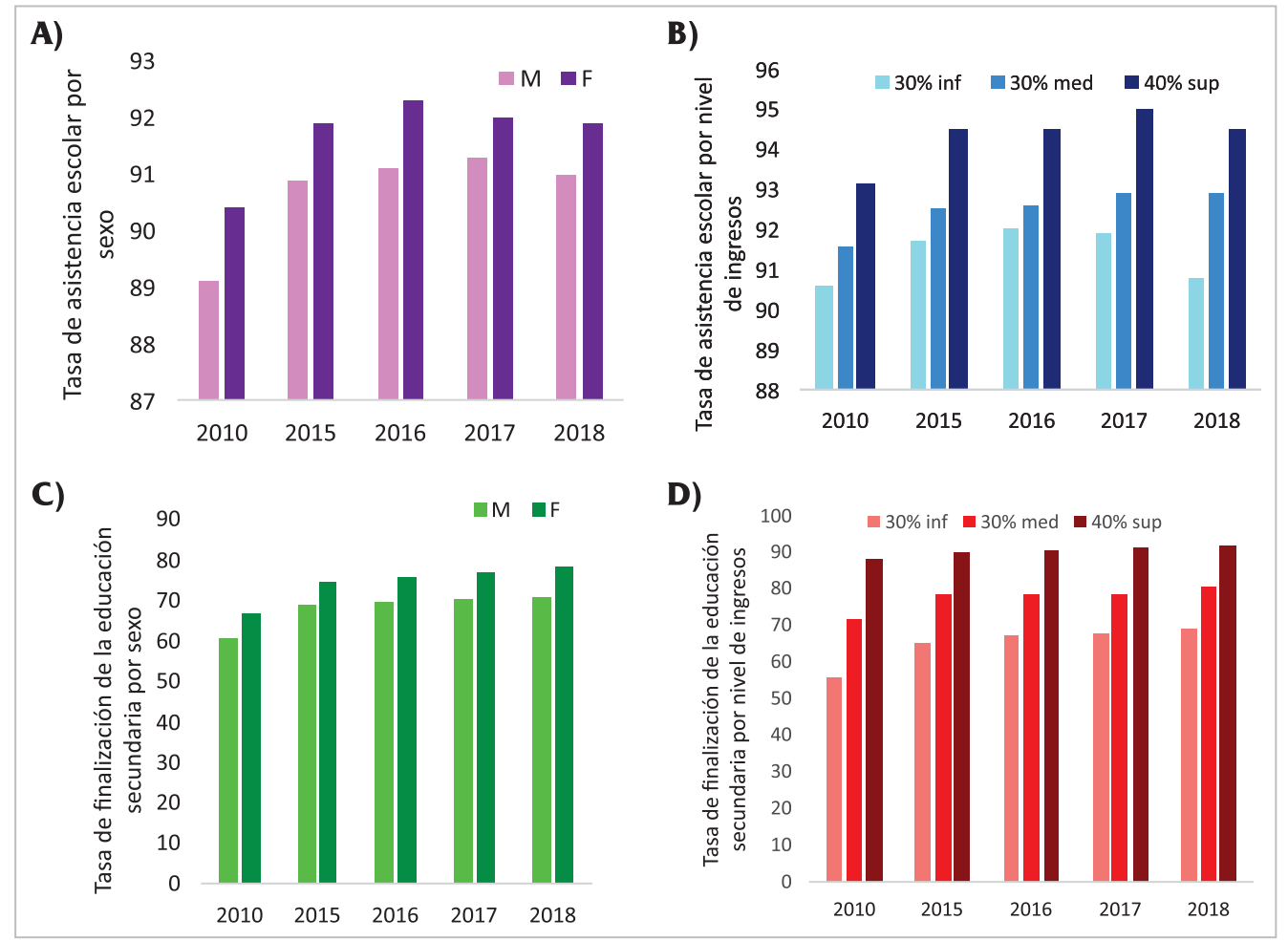

Esto implica que se perpetúan algunas limitaciones donde se cuestiona el deber ser versus lo que realmente se hace (Dussel, 2004), esto es, se generan políticas, pero su aplicación no llega a ser contundente (Frigerio, Diker y Mendoza, 2009). Por ejemplo, si bien se ha dado un aumento en los programas de inclusión, en igual medida ha crecido la exclusión social, o bien sea que se le piden muchos resultados a un programa que pretende articular muchas cosas que finalmente no se logran (Frigerio, Diker y Mendoza, 2009).

El acceso, con relación al nivel de ingresos, mostró que un mayor nivel de ingresos tendió a estabilizar el acceso a través del tiempo, aunque este nivel de ingresos tuvo una leve dismunición al 2018 (figura 1B). Esto podría ser explicado a la luz de la creciente existencia y demanda del sistema educativo privado, bajo la premisa de mayor calidad (MinEducación, 2016). El acceso con ingresos más bajos tuvo una tendencia temporal al de acceso con ingresos altos. Esto último podría estar en función al limitado grado de efectividad de las políticas públicas enfocadas a los más pobres, puesto que es superado en el 2010.

En relación con la dimensión egreso, en general, a través del tiempo 
no se supera el $22 \%$, siendo la menor para el 2005 tanto en hombres (18\%) como en mujeres (20\%), aunque la tendencia temporal es a un mayor egreso de mujeres, contrario a lo observado en el acceso; esto último no necesariamente implica que sean las más mujeres que terminan estudios si se hiciera un análisis más detallado (figura 1C). Referido al egreso por condición económica de los hogares, de forma general no se aprecian cambios sustanciales a través de los años. Pero ocurre una tendencia al porcentaje de personas que egresan a medida que pasa el tiempo en grupos familiares con mejor economía, incluso los que tendría menores ingresos los superan para el año 2010. El egreso se ve claramente favorecido en la población con ingresos medios, opuesto a lo hallado en relación con la dimensión de acceso, aunque no se mostraron fuertes las distancias en ningún año de análisis (figura 1D).

Entonces, Colombia aprecia avances en su universalización escolar, similar a otros tiempos y lugares en AL (Gentili, 2009), pero no se logra ni el acceso y dista muchos más del egreso educativo de toda la población. Además, a esta estadística se escapan datos de niños y jóvenes, u otras edades, que asisten en otros espacios educativos, llamados por algunos como "Escuela de segunda oportunidad" o escuelas populares alternativas (Spivak, 2010), que buscan abarcar a todos por igual, desprotegidos y en condiciones más favorables que, además, rompe con la sistematicidad de la educación que ha imperado por décadas, con mayor afianzamiento en AL, al propiciar alternativas pedagógicas y modificando el arte de enseñar (Meirieu, 1998), en la búsqueda de movilizar a los sujetos vistos de siempre como estáticos a sujetos que incorporan de forma válida sus saberes, formas y experiencias de vida sociales y culturales, con lo cual se da una ruptura de estereotipos (Arditi, 2009) que se han dado por hecho a través del tiempo. Dichos espacios, además, posibilitan que los sujetos (docente-estudiante) se reconozcan como diferentes, y se respeten mutualmente dichas identidades (Bellei et al., 2012).

En cuanto al resultado por ingresos, lo que se aprecia es que Colombia se ajusta al estado mundial, incompleto acceso y bajo egreso en relación con los más pobres y de mediano ingreso (Ortiz y Cummins, 2012). Así, la desigualdad se reproduce y se marca en clases sociales, pues ya hace más de 60 años se declaró la educación y la igualdad como un derecho; pero, como afirman estos autores, a ese ritmo hacen falta 800 años para que los más pobres logren un $10 \%$ de ingreso mundial. Sin duda, un análisis más fino podría visibilizar realidades y percibir puntos de inflexión de las zonas y brechas específicas, como lo muestran García y Quiroz (2011), tanto en estudiantes de clase baja y media-baja, que estudian en colegios públicos, tienen al final un menor número de años de educación y peores rendimientos académicos; como estudiantes de clase media-alta y alta, que estudian en 
colegios privados, tienen mayores años de educación y mejor desempeño en las pruebas oficiales.

Sin embargo, como lo hemos visto a lo largo del escrito desde el contexto, acceso y resultados, el camino de la calidad en educación podría tornase largo y complejo si no se analizan estos indicadores; de esta manera, se puede proyectar un escenario futuro a través de instituciones que adquieran el compromiso con las transformaciones, entre ellas, las del mercado laboral, ya que el informe del MEN/OCDE (2016) sugiere que "los estudiantes que continúan sus estudios después de terminar su educación básica secundaria tienen mejores salarios y una mayor posibilidad de ser empleados en la economía formal que aquellos que no obtuvieron un título de bachiller" (p. 34).

En síntesis, el balance en materia de resultados es positivo, pero aún quedan algunas cuentas pendientes y brechas por cerrar, entre ellas: la brecha regional, la brecha entre niveles de educación y la brecha socioeconómica. Estas brechas ya sugieren, de entrada, un rasgo del sistema educativo colombiano: la profunda desigualdad (García et al., 2013).

Dentro de esta disertación desde el contexto y el acceso, hacemos mención a la dimensión de resultados, estos permiten constatar que la educación básica secundaria sigue siendo el nivel de enlace más débil del sistema educativo en Colombia, con una "tasa de deserción anual del $4,5 \%$, superior a la de educación primaria $(3,2 \%)$ y educación media $(3,1 \%)$ [...] Solo el $30 \%$ de los jóvenes hace la transición de la escuela o colegio a la educación superior" (Ministerio de Educación Nacional/OCDE, 2016), sin mencionar que en ocasiones la educación básica y media se sigue centrando en un enfoque tradicional de conocimientos, sin tener presente el dinamismo y los cambios de la sociedad.

\section{CONCLUSIONES}

Colombia, año tras año, ha venido generando esfuerzos para fortalecer sus instituciones educativas y promover una gestión centrada en disminuir la desigualdad en sus múltiples dimensiones, pero aún quedan retos significativos respecto a los indicadores de "contexto", "acceso" $y$ "resultados". El indicador de "contexto" en el informe del OCDE (2016), evidencia que la pobreza y desigualdad siguen siendo retos del Sistema Educativo Colombiano, pues a pesar de los avances en el mejoramiento su eficacia, persisten desigualdades notorias entre regiones y estudiantes de zonas rurales y urbanas. Desde el SITEAL, personas con mayores ingresos han tendido constante finalización de estudios de educación media (90\%), mientras que esta finalización aumenta solo levemente para personas con ingresos inferiores. Por tanto, un progreso en los ingresos significaría mejora en la desigualdad educativa. Igualmente, el "acceso" a la educación ha sido una prioridad a través de políticas ambiciosas que buscan incrementar el número de estudiantes matriculados en todos 
los niveles y llevar los servicios educativos a todos los rincones del país, particularmente apuntando a la matrícula de más mujeres a través del tiempo. Sin embargo, la permanencia y continuidad en Primera infancia, Básica, Media y Educación Superior siguen siendo brechas considerables que existen en Colombia. Igualmente, ocurre con el ingreso tardío y los índices de repetición por grados. El sistema educativo ha pasado de poner su énfasis en la autonomía y cobertura, a centrarse en la calidad, la que notoriamente se evidencia en educación privada que requiere altos ingresos en las personas. Entonces, se requiere una revisión de las políticas que se ocupan de los ingresos en la sociedad, para evitar que sean una limitante de acceso a la educación. Respecto a los "resultados", quedan retos asociados con el aprendizaje, los resultados y el egreso, elementos claves en la calidad educativa. Es menester continuar avanzado y avizorando la forma en que Colombia puede mejorar la calidad y pertinencia de su sistema educativo, con el fin de alcanzar la meta de ser el país "mejor educado" de América Latina para el año 2025. 


\section{REFERENCIAS}

Arditi, B. (2009). Identidades metaestables: El destino del nómada. En La política en los bordes del liberalismo: diferencia, populismo, revolución, emancipación (pp. 29-38). Gedisa Editorial.

Asamblea General de las Naciones Unidas. (1948). Declaración Universal de los Derechos Humanos. París.

Asamblea Nacional Constituyente. (1991). Constitución Política de la República de Colombia. Bogotá, D.C.: Gaceta Constitucional No. 116 de 20 de julio de 1991 .

Atorresi, A., Mekler, V., y Feldman, D. (2012). Oportunidades para aprender. Sistematización de Programas en Argentina, Brasil, Chile, Colombia y México. UNICEF - Universidad Nacional General Sarmiento.

Bellei, C., Levinson, B., Pereira, G., Mauger, G., Sarramona, J., Tenti, E., ... Tiramonti, G. (2012). La escolarización de los adolescentes: desafíos culturales, pedagógicos y de política educativa. (E. Tenti, Ed.). Instituto Internacional de Planeamiento de la Educación IIPE - UNESCO.

Bellei, C., Poblete, X., Sepúlveda, P., Orellana, V., y Abarca, G. (2013). Situación educativa de América Latina y el Caribe: Hacia la educación de calidad para todos al 2015. (C. Bellei, Ed.). OREALC/UNESCO.

Bolívar, A. (2005). Equidad Educativa y teorías de la justicia. REICE. Revista Iberoamericana Sobre Calidad, Eficacia y Cambio En Educación, 3(2), 42-69. https://www.redalyc.org/pdf/551/55103205.pdf

Botinelli, M. (2016). Clase: La práctica del análisis de la desigualdad mediante indicadores educativos. In Seminario Desigualdades educativas en América Latina y el Caribe: Desafios actuales en prospectiva a una mayor justicia educativa. CLACSO/UNIPE.

Cecchini, S., Cuenca, R., Feijoó, M., García-Huidobro, J., González, M., Marco, F., ... Lobato, C. (2014). Educación y políticas sociales. Sinergias para la inclusión. (M. Feijoó y M. Poggi, Eds.). Instituto Internacional de Planeamiento de la Educación IIPE - UNESCO.

Congreso de la República de Colombia. (1994). Ley 115 de 1994. Por la cual se expide la ley general de educación. Bogotá, D.C.: Diario Oficial No. 41.214 de 8 de febrero de 1994.

Congreso de la República de Colombia. (2001). Ley 715 de 2001. Por la cual se dictan normas orgánicas en materia de recursos y competencias de conformidad con los artículos 151, 288, 356 y 357 (Acto Legislativo 01 de 2001) de la Constitución Política y se dictan otras disposiciones para organizarla . Bogotá, D.C.: Diario Oficial Año CXXXVII, No. 44.654 del 21 de diciembre de 2001 . 
Congreso de la República de Colombia. (2013). Ley Estatutaria 1618 de 2013. Por medio de la cual se establecen las disposiciones para garantizar el pleno ejercicio de los derechos de las personas con discapacidad. Bogotá, D.C.: Diario Oficial No. 48.717 de febrero 27 de 2013.

Delgado, M. (2014). La educación básica y media en Colombia: Retos en equidad y calidad. Informe final. Fedesarrollo.

Departamento Nacional de Planeación. (2007). Documento Conpes Social 109. Política pública nacional de primera infancia. "Colombia por la Primera Infancia”. Bogotá, D.C.

Dussel, I. (2004). Desigualdades sociales y desigualdades escolares en la Argentina de hoy. Algunas reflexiones y propuestas.

Frigerio, G., Diker, G., y Mendoza, S. (2009). Los proyectos de inclusión educativa y la problemática de su evaluación. En Programa EURO social Educación, Proyecto "Indicadores y procedimientos para el monitoreo y evaluación de proyectos de inclusión y promoción educativa” Centro de estudios multidisciplinarios.

García, M., Espinosa, J., Jiménez, F., y Parra, J. (2013). Separados y desiguales. Educación y clases sociales en Colombia. Centro de Estudios de Derecho, Justicia y Sociedad.

García, M., y Quiroz, L. (2011). Apartheid educativo: educación, desigualdad e inmovilidad social en Bogotá. Revista de Economía Institucional, 13(25), $137-162$.

Gentili, P. (2009). Marchas y Contramarchas y las dinámicas de exclusión incluyente en América Latina (a sesenta años de la Declaración Universal de los Derechos Humanos). Revista Iberoamericana de Educación, (49), 19-57.

Meirieu, P. (1998). Frankenstein educador. Laertes.

Ministerio de Educación. (2013). Sistema Nacional de Indicadores Educativos para los niveles de Preescolar, Básica y Media en Colombia. Bogotá DC, Colombia.

Ministerio de Educación Cultura y Deporte. (2014). Panorama de la educación. Indicadores de la OCDE 2014. Informe español. Madrid.

Ministerio de Educación Nacional/OCDE. (2016). Revisión de políticas nacionales de educación. La educación en Colombia. OECD/Ministerio Nacional de Educación.

Ministerio de Educación Nacional. (2003). Resolución 166 del 4 de 2003. Por medio de la cual se establecen las condiciones del reporte de información para la implementación de la primera etapa del Sistema de Información del Sector Educativo. 
Ministerio de Educación Nacional. (2006a). Plan Nacional de Educación 2006-2016.

Ministerio de Educación Nacional. (2006b). Resolución 5360 de 2006. Por la cual se organiza el proceso de matrícula oficial de la educación preescolar, básica y media en las entidades territoriales certificadas.

Morduchowicz, A. (2006). Los indicadores educativos y las dimensiones que los integran. UNESCO/IIPE-UNESCO.

OECD, United Nations, \& CAF. (2014). Latin American economic outlook 2015. Educations, skills and innovation for development. OECD - United Nations - CAF. https://doi.org/10.1787/leo-2015-en

ONU. (2015, septiembre). La Asamblea General adopta la Agenda 2030 para el Desarrollo Sostenible. Objetivos de Desarrollo Sostenible.

Ortiz, I., y Cummins, M. (2012). Desigualdad global: La distribución del ingreso en 141 países. UNICEF.

PNUD. (2015). Panorama general. Informe sobre Desarrollo Humano 2015. Trabajo al servicio del desarrollo humano. PNUD.

Presidencia de la República de Colombia. (1978). Decreto 1419 de 1978. Por el cual se señalan las normas y orientaciones básicas para la administración curricular en los niveles de educación preescolar básica (primaria y secundaria), media vocacional e intermedia profesional. Bogotá, D.C.: Diario Oficial No. 35070 del 8 de agosto de 1978.

Presidencia de la República de Colombia. (1997). Decreto 2247 de 1997. Por el cual se establecen normas relativas a la prestación del servicio educativo del nivel preescolar y se dictan otras disposiciones.

Presidencia de la República de Colombia. (2002). Decreto 1526 de 2002. Por el cual se reglamenta la administración del sistema de información del sector educativo. Bogotá, D.C.: Diario Oficial No. 44.833 del 24 de julio de 2002.

Presidencia de la República de Colombia. (2011). Decreto 4875 de 2011. Por el cual se crea la Comisión Intersectorial para la Atención Integral de la Primera Infancia - AIPI - y la Comisión Especial de Seguimiento para la Atención Integral a la Primera Infancia. Bogotá, D.C.: Diario Oficial No. 48.291 de 22 de diciembre de 2011.

Presidencia de la República de Colombia. (2017). Decreto 1421 de 2017. Por el cual se reglamenta en el marco de la educación inclusiva la atención educativa a la población con discapacidad.

Serra, M., y Canciano, E. (2006). Las condiciones de enseñanza en contextos críticos. Ministerio de Educación, Ciencia y Tecnología de la Nación - OEA. 
Spivak, G. (2010). Crítica de la razón poscolonial. Hacia una historia del presente. Akal.

Tedesco, J. (2010). La educación en el horizonte 2020. Educación y justicia: el sentido de la educación. Fundación Santillana.

Tiana, A. (1997). Qué son y qué pretenden. Cuadernos de Pedagogía, (256), 50-53.

Trucco, D. (2014). Educación y Desigualdad en América Latina. CEPAL. https://repositorio.cepal.org/bitstream/handle/11362/36835/1/S2014209_es.pdf

UNESCO. (2009). Indicadores de la educación Especificaciones técnicas. Unesco Institute for Statistics. http://uis.unesco.org/sites/default/files/documents/ education-indicators-technical-guidelines-sp.pdf 